\title{
Surface Green's function of a piezoelectric half-space
}

\author{
Vincent Laude, Carlos F. Jerez-Hanckes, and Sylvain Ballandras
}

\begin{abstract}
The computation of the two-dimensional harmonic spatial-domain Green's function at the surface of a piezoelectric half-space is discussed. Starting from the known form of the Green's function expressed in the spectral domain, the singular contributions are isolated and treated separately. It is found that the surface acoustic wave contributions, i.e. poles in the spectral Green's function, give rise to an anisotropic generalization of the Hankel function $H_{0}^{(2)}$, the spatial Green's function for the scalar two-dimensional wave equation. The asymptotic behavior at infinity and at the origin (for the electrostatic contribution) are also explicitely treated. The remaining non-singular part of the spectral Green's function is obtained numerically by a combination of fast Fourier transform and quadrature. Illustrations are given in the case of a substrate of Y-cut lithium niobate.
\end{abstract}

Index Terms-Green's functions, piezoelectric media, surface acoustic waves.

\section{INTRODUCTION}

Green's functions are extensively used in the numerical simulation of surface acoustic wave (SAW) transduction problems. Usually, in SAW problems, the Green's function relates the excitation at the origin in term of stresses and electrical charges to the response at another point of the surface in terms of mechanical displacements and electric potential [1], [2]. For instance, the finite element method / boundary element method (FEM/BEM) relies on a spatial-domain Green's function used to account for wave propagation in the substrate but also to describe the electrostatic response of the substrate. Such Green's functions can include the periodicity of the excitation, as in the case of the periodic FEM/BEM [3]-[9], which describes the propagation of waves in an infinite periodic electrode grating. Because of the use of Floquet waves in the periodic case, it turns out that it is the spectral-domain Green's function that is needed. This is not the case of the so-called finite twodimensional FEM/BEM, in which the full one-dimensional (1D) spatial Green's function is used [10]. Even though the finite FEM/BEM can describe accurately the response of a finite length SAW device, it cannot take into account the transverse dimension, and hence misses important phenomena such as diffraction and wave guiding effects, or more subtle transverse effects such as the generation of spurious surface waves in the region between electrodes and buses [11]. Many approximate models have been proposed to handle transverse

The authors are with the département LPMO of the Institut FEMTOST, CNRS UMR 6174, 32 avenue de l'Observatoire, F-25044 Besançon, France (e-mail: vincent.laude@femto-st.fr). Carlos Jerez-Hanckes is also with the Centre de Mathématiques Appliquées, CNRS UMR 7641, Ecole polytechnique, F-91128 Palaiseau, France, and with Temex, Sophia-Antipolis, F-06904, France. and diffraction effects (see e.g. Refs. [12]-[17]). However, a rigorous description should encompass all wave generation, propagation and detection phenomena together with electrostatic effects within the quasi static approximation [18], [19]. The spatial Green's function of the surface of a piezoelectric half-space is in this sense the most elementary yet complete description of three-dimensional effects.

The computation of the spatial Green's function of the surface of a piezoelectric half-space relies on the Fourier transform of the spectral Green's function. The spectral Green's function is obtained by assuming plane wave propagation with a given wavevector in the plane of the surface, and solving for the wavevector component along the axis entering the substrate. Hence, it inherently includes a complete threedimensional description. In this work, we consider the computation of the full two-dimensional surface Green's function of a piezoelectric half-space. By two-dimensional we mean that the spatial variables are considered along the surface with the third dimension (orthogonal to the surface) implicitly included. We consider this problem as a necessary step towards the generalization of two-dimensional FEM/BEM algorithms to three-dimensional FEM/BEM algorithms. As remarked above, what is basically needed is to compute the two-dimensional Fourier transform of the spectral surface Green's function of the piezoelectric half-space. However, this is not straightforward as the most interesting features of the problem arise as singularities of the spectral Green's function. For instance, the surface acoustic waves themselves contribute as poles, while bulk acoustic waves contribute less singularly as discontinuities of the first derivative. Furthermore, the asymptotic behaviors at infinity and at the origin must also be treated with care. In particular, the $1 / s$ singularity at the origin only appears in the electrostatic part of the problem.

In Section II, we recall how the spectral Green's function can be computed efficiently and we discuss the form of the different singular contributions. In Section III, we isolate the case of poles, i.e. SAW contributions at infinity in the spatial domain, and show how the well-known two-dimensional spatial isotropic Green's function is generalized in the case of anisotropy. In Section IV, we briefly discuss the case of asymptotic contributions and give analytic expressions for them. In Section V, we propose an efficient algorithm to obtain the non-singular part of the Fourier transform and gather up the different contributions that make up the 2D spatial Green's function. 


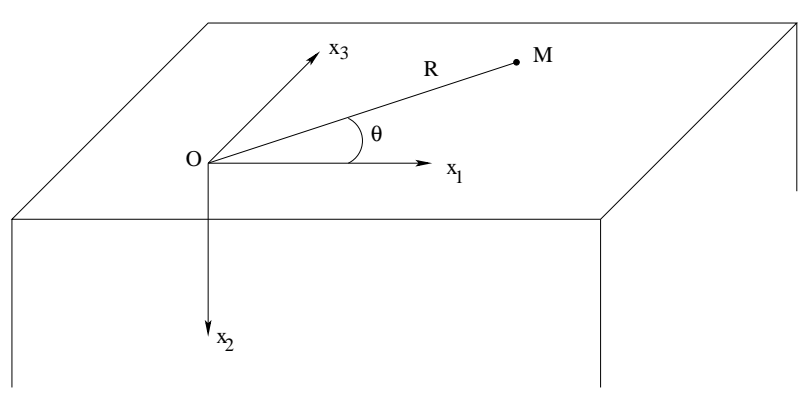

Fig. 1. Definition of axes and coordinates for the computation of the spatial Green's function of a piezoelectric half-space.

\section{SPECTRAL GREEN's FUNCTION}

The most usual form of the spectral Green's function, or spectral Green's dyadic, for a piezoelectric half-space is in the form of a linear relation between generalized displacements and stresses [20]. The generalized displacements include the mechanical displacements $u_{1}, u_{2}, u_{3}$, and the electrical potential as $u_{4}=\phi$. The generalized stresses include the mechanical stresses applied to the surface $T_{21}, T_{22}, T_{23}$, and the electrical displacement normal to the surface as $T_{24}=D_{2}$. Throughout this paper, we assume a time-harmonic dependence of the form $\exp (\imath \omega t)$. The notations for axes are given in Fig. 1. Solving the problem of plane wave propagation with given slownesses $s_{1}$ and $s_{3}$ along the surface, a linear relation between generalized displacements and stresses is found as

$$
\left(\begin{array}{c}
\tilde{u}_{1} \\
\tilde{u}_{2} \\
\tilde{u}_{3} \\
\tilde{u}_{4}
\end{array}\right)=\frac{\tilde{G}\left(s_{1}, s_{3}\right)}{-\imath \omega}\left(\begin{array}{c}
\tilde{T}_{21} \\
\tilde{T}_{22} \\
\tilde{T}_{23} \\
\tilde{T}_{24}
\end{array}\right),
$$

where we use the tilde over a field, e.g. $\tilde{u}_{1}$, to emphasize that the quantity is considered in the spectral domain rather than in the spatial domain. In this equation, $\tilde{G}$ is a square matrix of functions of dimension 4, termed the spectral Green's function. Various practical methods for the computation of the spectral Green's function of a piezoelectric half-space have been given in the literature [1], [2], [21], [22]. We assume in the following that $\tilde{G}$ can be obtained efficiently and with great precision.

In this paper, we shall be concerned with obtaining the surface Green's function $G$ in the spatial domain, i.e. the two-dimensional Fourier transform of $\tilde{G}$. The spatial-domain Green's function of a piezoelectric half-space is here defined as the generalized displacements response to a time-harmonic generalized stress excitation located at the origin. From the spectral Green's function, the spatial Green's function at the point of the surface with polar coordinates $(R, \theta)$ is obtained as the two-dimensional Fourier transform

$$
G(R, \theta)=\int_{0}^{\infty} \frac{k \mathrm{~d} k}{(2 \pi)^{2}} \int_{0}^{2 \pi} \mathrm{d} \psi \tilde{G}(k, \psi) e^{-\imath k R \cos (\psi-\theta)}
$$

This formula follows from the plane wave expansion of the exciting delta function,

$$
\begin{aligned}
\delta\left(x_{1}, x_{3}\right) & =\int_{-\infty}^{\infty} \int_{-\infty}^{\infty} \frac{\mathrm{d} k_{1} \mathrm{~d} k_{3}}{(2 \pi)^{2}} e^{-\imath\left(k_{1} x_{1}+k_{3} x_{3}\right)} \\
& =\int_{0}^{\infty} \frac{k \mathrm{~d} k}{(2 \pi)^{2}} \int_{0}^{2 \pi} \mathrm{d} \psi e^{-\imath k R \cos (\psi-\theta)}
\end{aligned}
$$
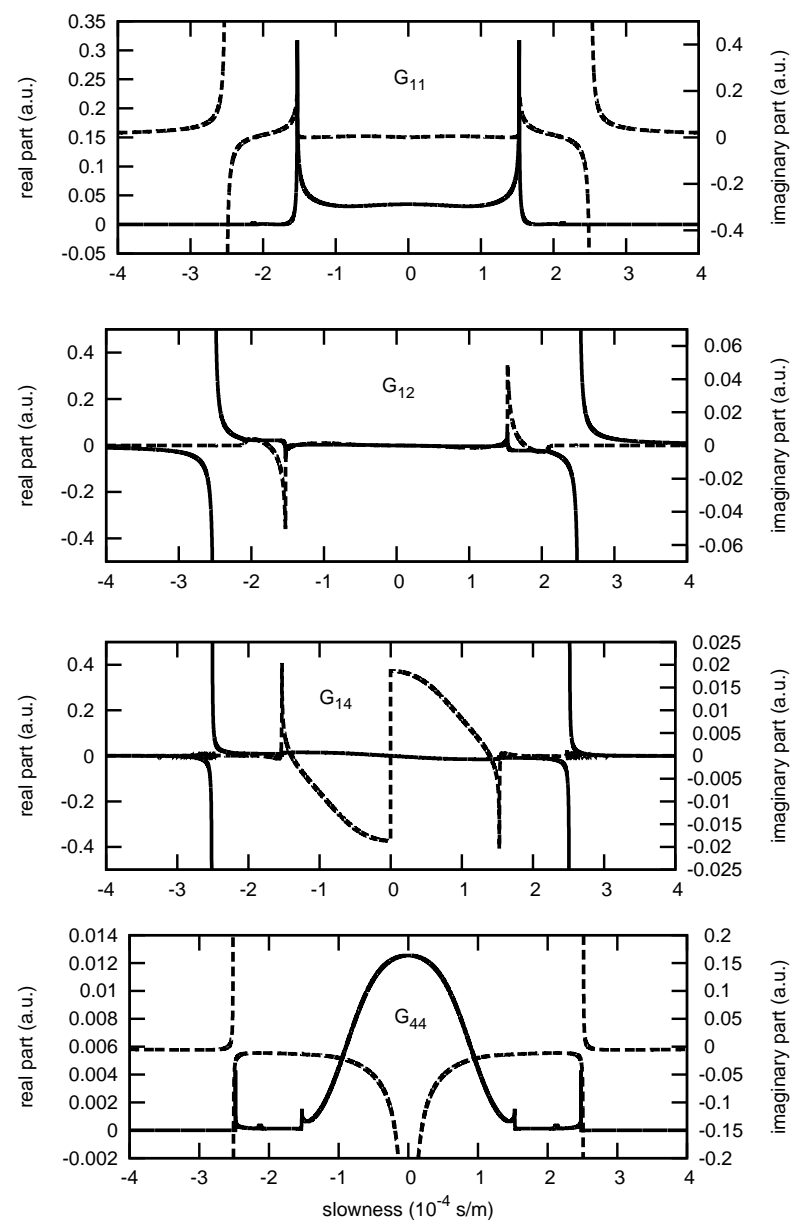

Fig. 2. Cross sections of four of the spectral Green's function components as a function of slowness for $\mathrm{Y}+128$ lithium niobate and $\mathrm{X}$ propagation. The real (solid line) and imaginary (dashed line) parts are shown.

In the above equations, the wave vector $\mathbf{k}$ is linked to the slowness vector $\mathbf{s}$ by $\mathbf{k}=\omega \mathbf{s}$. Furthermore, $k_{1}=k \cos (\psi)$ and $k_{3}=k \sin (\psi)$.

It is not possible to use directly a numerical algorithm like the fast Fourier transform (FFT) because $\tilde{G}$ has singularities in the spectral plane. Consider the plots in Fig. 2 which displays some representative components of $\tilde{G}$ in the particular case of a $\mathrm{Y}+128$ cut lithium niobate half-space as cross sections along the crystallographic $\mathrm{X}$ axis. All cross sections exhibit two poles placed symmetrically with respect to the origin which are the signature of the surface acoustic wave. We model this pole by a function of the form

$$
\tilde{G}_{s}(k, \psi)=a_{s}(\psi) \frac{k_{0}^{2}}{k^{2}-k_{s}^{2}(\psi)},
$$

with $k_{s}(\psi)=\omega s_{s}(\psi)$ where $s_{s}(\psi)$ is the slowness of the SAW in the direction given by $\psi$ obtained on a stress-free surface with open-circuited condition. $k_{0}$ is an arbitrarily chosen wave vector used only for normalization purposes. $a_{s}(\psi)$ amounts for the anisotropy of the electromechanical coupling of the SAW and has the same units as the considered spectral Green's function component. Note that even though the SAW slowness satisfies the central symmetry property $s(\psi)=s(\psi+\pi)$, such 


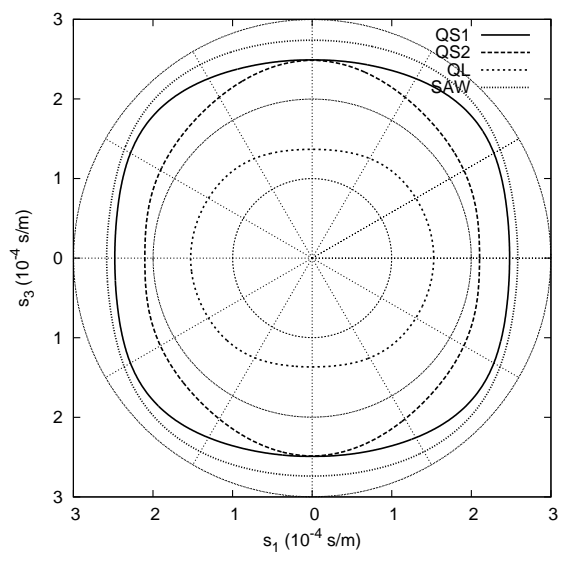

Fig. 3. Slowness curves for the surface acoustic wave (SAW) and the bulk acoustic waves (QS1, QS2, QL) propagating in the plane of a Y+128 lithium niobate half-space. QS1 and QS2 stand for the two quasi shear bulk acoustic waves, while QL stands for the quasi longitudinal bulk acoustic wave.

is not necessarily the case for $a_{s}(\psi) . s(\psi)$ and $a_{s}(\psi)$ can be estimated using fit procedures [15].

There is an additional pole at the origin only in the case of $\tilde{G}_{44}$, i.e. for the electrostatic part of the spectral Green's function only. This pole can be interpreted as arising from the singularity due to an infinite line charge on the surface and is modelled as

$$
\tilde{G}_{0}(k, \psi)=a_{0}(\psi) \frac{k_{0}}{k} .
$$

The symmetry relation $a_{0}(\psi+\pi)=a_{0}(\psi)$ holds. $a_{0}(\psi)$ can be obtained as the limit of $k \tilde{G}_{44} / k_{0}$ as $k$ tends to zero. The asymptotic behavior at infinity for all Green's function components is also governed by a slow $1 / k$ decrease which we model as

$$
\tilde{G}_{\infty}(k, \psi)=a_{\infty}(\psi) \frac{k_{0} k}{k_{0}^{2}+k^{2}} .
$$

This form is chosen such as not to introduce a pole at the origin while still having the required $1 / k$ asymptotic behavior at infinity. In the case of $\tilde{G}_{44}$, the value of $a_{\infty}(\psi)$ includes a correction equal to $-a_{0}(\psi)$ since Eq. (5) introduces a spurious $1 / k$ asymptotic behavior at infinity. The symmetry relation $a_{\infty}(\psi+\pi)=a_{\infty}(\psi)$ holds. $a_{\infty}(\psi)$ can be obtained as the limit of $k \tilde{G}_{i j} / k_{0}$ as $k$ tends to infinity.

A discontinuity at the origin is seen to exist in the case of $\tilde{G}_{14}$. It will be seen later that it is not necessary to model explicitely this form of contribution. Discontinuities of the first derivative of the functions are seen to occur at definite slownesses. These slownesses correspond to bulk acoustic waves propagating in the sagittal plane with their Poynting vector oriented in the direction $\psi$, the so-called surface skimming bulk waves (SSBW). All these features need to be taken into account in the Fourier transform. Clearly, the surface wave contribution is the most important in the far field where it is expected to be dominant. Bulk waves are classically expected to decrease faster with increasing distance from the origin of the excitation [1]. It is important to note that though the discontinuities can be sharp, their dependence with the direction of propagation is always smooth. Fig. 3

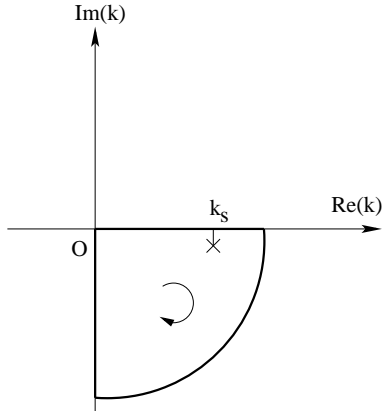

(a)

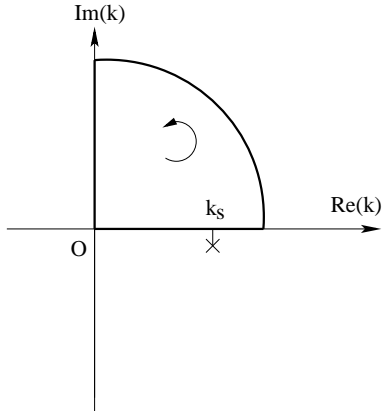

(b)
Fig. 4. Contours for integration in the complex $k$-plane. (a) Contour used when $R \cos (\psi-\theta)>0$. (b) Contour used when $R \cos (\psi-\theta)<0$. The pole $k_{s}$ due to the surface acoustic wave is displaced downwards vertically from the real axis.

displays the slowness curves for the pole created by the surface acoustic wave and for the bulk acoustic waves within a Y+128 cut lithium niobate half-space. The essential features of these slowness curves is that they are very smooth and periodic. These properties will be exploited in the following derivations.

The surface Green's function will be computed by separating the different singular contributions and the regular part of the spectral Green's function. It takes the form

$$
G(R, \theta)=G_{s}(R, \theta)+G_{0}(R, \theta)+G_{\infty}(R, \theta)+G_{n s}(R, \theta),
$$

where $G_{s}, G_{0}, G_{\infty}$ and $G_{n s}$ account for the SAW contribution, the electrostatic contribution, the asymptotic contribution at infinity and the non-singular contribution, respectively.

\section{SURFACE ACOUSTIC WAVE CONTRIBUTION}

The SAW contribution to the spectral Green's function assumes the form of Eq. (4). The spatial contribution to the Green's function is then

$$
\begin{aligned}
G_{s}(R, \theta)= & \frac{k_{0}^{2}}{(2 \pi)^{2}} \int_{0}^{2 \pi} a_{s}(\psi) \mathrm{d} \psi \\
& \int_{0}^{\infty} k \mathrm{~d} k \frac{e^{-\imath k R \cos (\psi-\theta)}}{k^{2}-k_{s}^{2}(\psi)},
\end{aligned}
$$

where we explicitely take the $k$-integral before the $\psi$-integral. The $k$-integral is singular and before it can be evaluated by the residue theorem the poles must be displaced slightly from the real axis. Such a procedure is usual in textbooks dealing with Green's functions of the wave equation and can be derived from the principle of limiting absorption [23]. The rule is that the pole at $+k_{s}(\psi)$ must be displaced downwards vertically in the complex plane, while the pole at $-k_{s}(\psi)$ must be displaced upwards vertically. The integration contours in the complex $k$-plane are indicated in Fig. 4 . The result of the integration depends on the sign of $R \cos (\psi-\theta)$. When $R \cos (\psi-\theta)>0$, the integration contour includes the SAW pole and then by application of the residue theorem

$$
\begin{array}{r}
\int_{0}^{\infty} k \mathrm{~d} k \frac{e^{-\imath k R \cos (\psi-\theta)}}{k^{2}-k_{s}^{2}(\psi)}=h\left(k_{s}(\psi) R \cos (\psi-\theta)\right) \\
-\imath \pi \exp \left(-\imath k_{s}(\psi) R \cos (\psi-\theta)\right)
\end{array}
$$




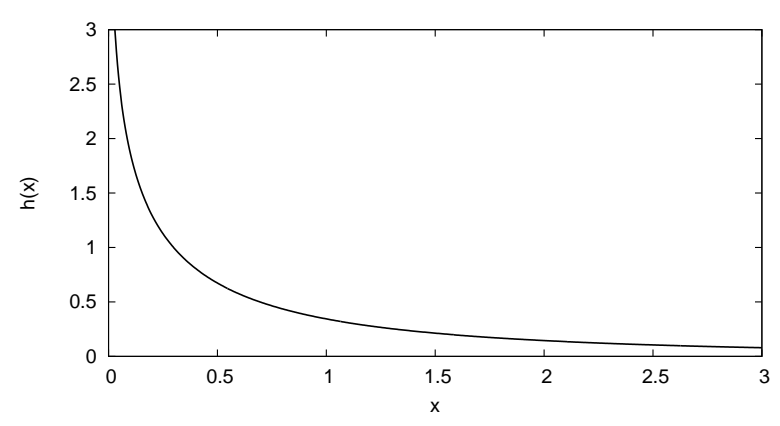

Fig. 5. Plot of function $h(x)$ defined by Eq. (10).

with

$$
h(x)=\int_{0}^{\infty} y \mathrm{~d} y \frac{\exp (-y x)}{y^{2}+1} .
$$

This integral, which has the form of a Laplace transform, is rapidly converging for $x \neq 0$ though divergent at the origin. The function $h(x)$ is easily evaluated numerically by standard algorithms and is plotted in Fig. 5. For small $x$, $h(x) \approx-\log (x)-\mathcal{C}$ where $\mathcal{C}$ is the Euler constant, while $h(x) \approx 1 / x^{2}$ for large $x$. When $R \cos (\psi-\theta)<0$, the integration contour does not include the pole and

$$
\int_{0}^{\infty} k \mathrm{~d} k \frac{e^{-\imath k R \cos (\psi-\theta)}}{k^{2}-k_{s}^{2}(\psi)}=h\left(k_{s}(\psi) R|\cos (\psi-\theta)|\right) .
$$

Gathering up the results, we obtain that the SAW contribution to the spatial surface Green's function is

$$
\begin{aligned}
& G_{s}(R, \theta)=\frac{k_{0}^{2}}{(2 \pi)^{2}} \\
& \times\left(\int_{0}^{2 \pi} \mathrm{d} \phi a_{s}(\phi+\theta) h\left(k_{s}(\phi+\theta) R|\cos \phi|\right)\right. \\
&\left.-\imath \pi \int_{-\pi / 2}^{\pi / 2} \mathrm{~d} \phi a_{s}(\phi+\theta) e^{\left.-\imath k_{s}(\phi+\theta) R \cos \phi\right)}\right),
\end{aligned}
$$

with $\phi=\psi-\theta$. This formula generalizes the scalar case as is easily seen by inserting $k_{s}(\psi)=k_{i}$ and $a_{s}(\psi)=a_{i}$ which, as outlined in the Appendix, results in $G_{s}(R, \theta)=$ $-\imath\left(k_{0}^{2} a_{i} / 4\right) H_{0}^{(2)}\left(k_{i} R\right)$, i.e. that in this case the spatial Green's function is the Hankel function of zero-th order and second kind, as it should [24]. In other words, we have found an integral representation of the SAW contribution to the anisotropic spatial Green's function that generalizes naturally the wellknown isotropic case. It is remarkable that the angular integral involves solely the SAW slowness curve, which stresses the intimate relationship between the slowness curve and the far field behavior of waves in anisotropic media. The first integral in Eq. (12) is an expansion over evanescent waves which has non negligible values only in the near field, i.e. close to the origin. The second integral is an expansion over plane waves and is dominant in the far field. Only one half of the slowness curve is involved in the superposition at the observation point. The involved plane waves can be viewed physically as the only
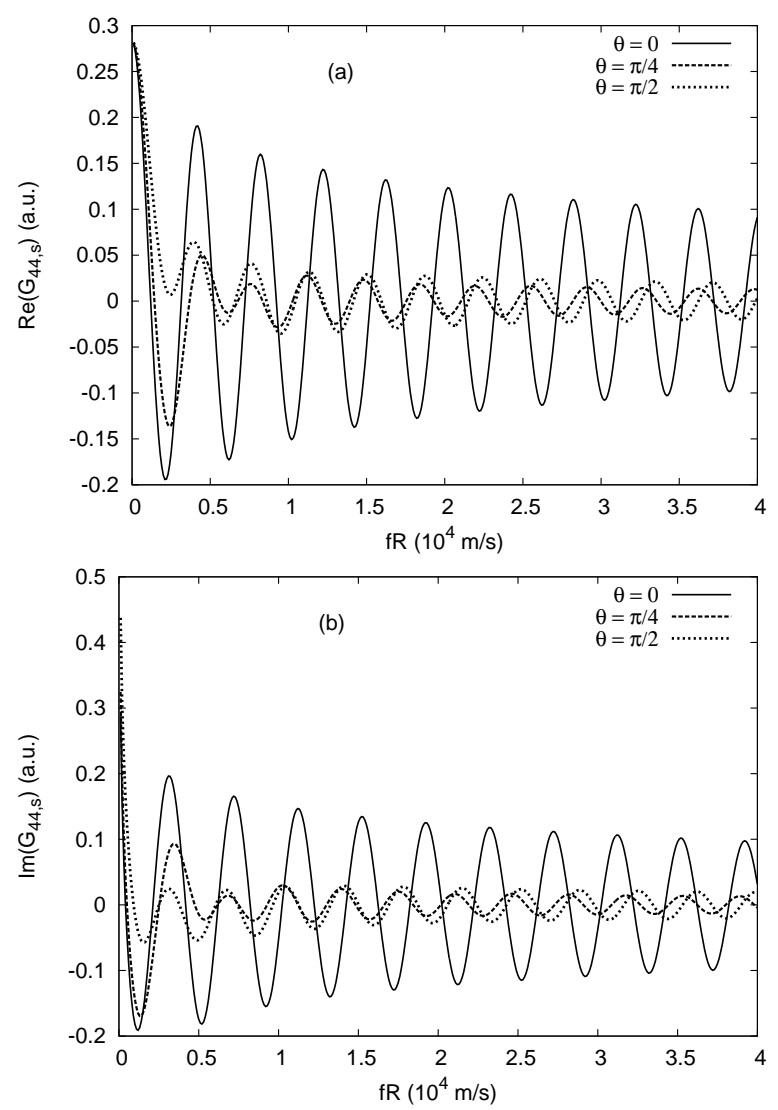

Fig. 6. Real (a) and imaginary (b) parts of $G_{44, s}$, the SAW contribution to the spatial surface Green's function $G_{44}$, for Y+128 lithium niobate.

outgoing plane waves emitted at the origin that encounter the observation point.

Fig. 6 displays the SAW contribution to the spatial surface Green's function $G_{44}$ computed for a Y+128 lithium niobate half-space, for three different propagation directions. It can be seen that this function has an oscillatory behavior that resembles that of Bessel functions. In particular, the envelope of the oscillations decreases as $1 / \sqrt{R}$ as $R$ increases. The near field disappears rapidly within a few oscillations. It is also seen that the amplitude of the oscillations depends rather strongly on the propagation direction, indicating that the surface Green's function is rather strongly anisotropic. This last point can be emphasized by further observing that the oscillations on the surface concentrate along the wave surface. To see this, the stationary phase principle can be applied to the plane wave expansion in Eq. (12) that is dominant in the far field. The stationary points of the integral are given as a function of the observation direction $\theta$ by the angles $\phi$ that satisfy $\left.\frac{\mathrm{d}}{\mathrm{d} \phi}\left(k_{s}(\phi+\theta) \cos \phi\right)\right)=0$. This last condition defines the power-flow angle from the slowness surface in anisotropic media.

The case of pseudo or leaky surface waves (LSAW) is also included in the result (12). Indeed, in this case the displacement of the poles from the real axis depicted in Fig. 4 is now not a mathematical artifice but the consequence of the appearance of an imaginary part of the slowness $s_{s}(\psi)$ [1], [25]-[27]. 


\section{ASYMPTOTIC BEHAVIOR CONTRIBUTION}

As explained in Section II, the asymptotic behavior at the origin of the spectral Green's function is of the form $a_{0}(\psi) k_{0} / k$. The asymptotic contribution to the spatial Green's function is then

$$
G_{0}(R, \theta)=\int_{0}^{\infty} \frac{k_{0} \mathrm{~d} k}{(2 \pi)^{2}} \int_{0}^{2 \pi} \mathrm{d} \phi a_{0}(\phi+\theta) e^{-\imath k R \cos \phi} .
$$

Since $a_{0}$ is a $2 \pi$-periodic function, we can insert its Fourier series representation

$$
a_{0}(\psi)=\sum_{n=-\infty}^{\infty} a_{0 n} \exp (\imath n \psi)
$$

in Eq. (13) to obtain

$$
G_{0}(R, \theta)=\frac{k_{0}}{2 \pi} \sum_{n=-\infty}^{\infty} \imath^{n} a_{0 n} \exp (\imath n \theta) \int_{0}^{\infty} J_{n}(k R) \mathrm{d} k,
$$

where the following integral representation of Bessel functions of integer order was used

$$
J_{n}(x)=\frac{1}{2 \pi \imath^{n}} \int_{0}^{2 \pi} \mathrm{d} y \exp (\imath(n y+x \cos y)) .
$$

Using the normalization of Bessel function

$$
\int_{0}^{\infty} J_{n}(x) \mathrm{d} x=(\operatorname{sgn}(n))^{n},
$$

where $\operatorname{sgn}(n)$ is the sign function that equals 1 if $n \geq 0$ and -1 otherwise, we finally have

$$
G_{0}(R, \theta)=\frac{k_{0}}{2 \pi R} \sum_{n=-\infty}^{\infty} \imath^{n}(\operatorname{sgn}(n))^{n} a_{0 n} \exp (\imath n \theta) .
$$

This contribution is easily obtained numerically from the Fourier coefficients of $a_{0}$. The $1 / R$ behavior at the origin is typical of electrostatic surface problems and the summation accounts for anisotropy.

As explained in Section II, the asymptotic behavior at infinity of the spectral Green's function is of the form $a_{\infty}(\psi) k_{0} k /\left(k_{0}^{2}+k^{2}\right)$. The asymptotic contribution to the spatial Green's function is then

$$
\begin{aligned}
G_{\infty}(R, \theta)= & \frac{k_{0}}{(2 \pi)^{2}} \int_{0}^{\infty} \frac{k^{2}}{k_{0}^{2}+k^{2}} \mathrm{~d} k \\
& \times \int_{0}^{2 \pi} \mathrm{d} \phi a_{\infty}(\phi+\theta) e^{-\imath k R \cos \phi} .
\end{aligned}
$$

Again, since $a_{\infty}$ is a $2 \pi$-periodic function, we can insert its Fourier series representation

$$
a_{\infty}(\psi)=\sum_{n=-\infty}^{\infty} a_{\infty n} \exp (\imath n \psi)
$$

in Eq. (19) to obtain

$$
G_{\infty}(R, \theta)=\frac{k_{0}}{2 \pi} \sum_{n=-\infty}^{\infty} \imath^{n} a_{\infty n} e^{\imath n \theta} \int_{0}^{\infty} \frac{k^{2} J_{n}(k R)}{k_{0}^{2}+k^{2}} \mathrm{~d} k .
$$

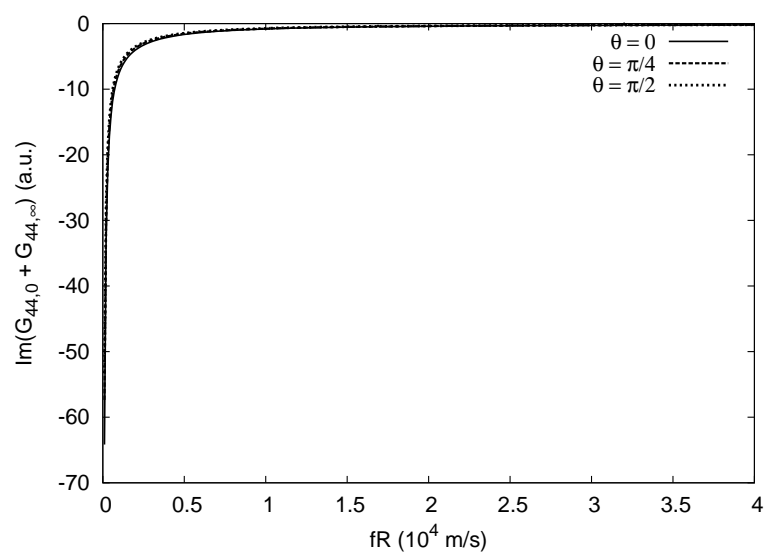

Fig. 7. Imaginary part of $G_{44,0}+G_{44, \infty}$, the asymptotic contributions to the spatial surface Green's function $G_{44}$, for Y+128 lithium niobate.

Using Eq. (17), we finally have

$$
\begin{aligned}
G_{\infty}(R, \theta)= & \frac{k_{0}^{2}}{2 \pi} \sum_{n=-\infty}^{\infty} \imath^{n} a_{\infty n} \exp (\imath n \theta)\left(\frac{(\operatorname{sgn}(n))^{n}}{k_{0} R}\right. \\
& \left.-\int_{0}^{\infty} \frac{J_{n}\left(y k_{0} R\right)}{1+y^{2}} \mathrm{~d} y\right)
\end{aligned}
$$

The integral on the right-hand side of the last equation can easily be evaluated by quadrature.

Fig. 7 displays the imaginary part of the asymptotic contributions to the spatial surface Green's function $G_{44}$ for $\mathrm{Y}+128$ lithium niobate half-space, again for three different propagation directions. Note that $a_{0}$ and $a_{\infty}$ are both purely imaginary. It is seen that the asymptotic contributions are mostly localized in the near field and exhibit a $1 / R$ dependence at the origin. The anisotropy is very weak in comparison with the SAW amplitude shown in Fig. 6.

\section{NON-SINGULAR PART CONTRIBUTION}

The singular contributions discussed above can be efficiently removed from the spectral Green's function by fitting to the models in Eqs. (4-6). The result of this procedure is depicted in Fig. 8 for the three propagation directions already used in plotting Figs. 6 and 7. It can be seen that there remains mostly smooth variations except at the slownesses of the SSBW where the derivative can be discontinuous, though the function remains continuous. Only in the direction $\theta=\pi / 2$ can a LSAW contribution be seen to remain. This contribution is due to a weak longitudinally polarized LSAW. However, due to its losses, it does not vary too abruptly and the non-singular spectral Green's function can be sampled at a moderate rate along the slowness axis.

The contribution of the non-singular part of the spectral Green's function is

$$
\begin{aligned}
G_{n s}(R, \theta)= & \int_{0}^{2 \pi} \mathrm{d} \psi \int_{0}^{\infty} \frac{k \mathrm{~d} k}{(2 \pi)^{2}} \\
& \times \tilde{G}_{n s}(k, \psi) e^{-\imath k R \cos (\psi-\theta)} .
\end{aligned}
$$

This integral is of course non-singular but it remains to be evaluated efficiently. A possibility would be to switch back 

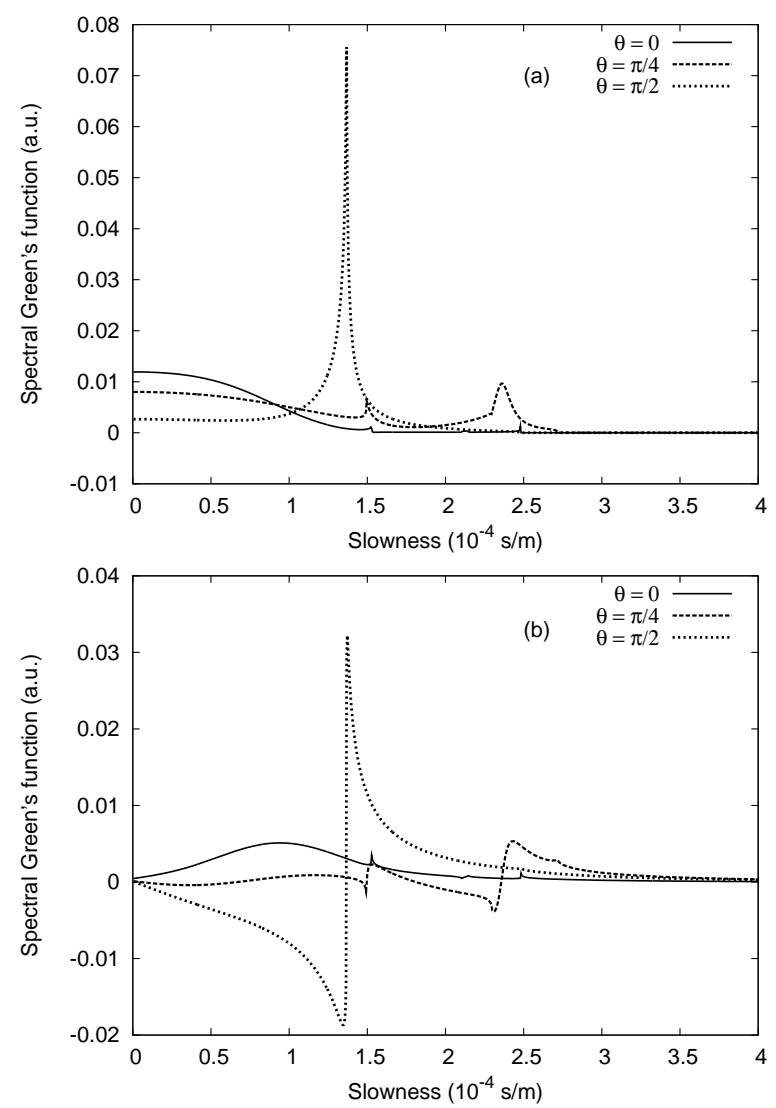

Fig. 8. Real (a) and imaginary (b) parts of the spectral surface Green's function $G_{44}$ with the SAW pole and the asymptotic contributions removed, for $\mathrm{Y}+128$ lithium niobate.

to Cartesian coordinates and take a two-dimensional fast Fourier transform (2D FFT) of the spectral Green's function. The practical application of this procedure is limited by the sampling requirement along the $k$-axis. Indeed, $\tilde{G}_{n s}(k, \psi)$ is a relatively fast varying function of $k$ but slowly varying function of $\psi$. Using Cartesian coordinates means that the sampling along both axes $k_{1}$ and $k_{3}$ must be as fine as it would be along the radial $k$ axis. Instead, we prefer to use a one-dimensional FFT along $k$ and a simple quadrature formula along $\psi$. More precisely we transform Eq. (23) above to

$$
\begin{aligned}
G_{n s}(R, \theta)= & \int_{-\pi / 2}^{\pi / 2} \mathrm{~d} \psi \int_{-\infty}^{\infty} \frac{|k| \mathrm{d} k}{(2 \pi)^{2}} \\
& \times \tilde{G}_{n s}(k, \psi) e^{-\imath k R \cos (\psi-\theta)},
\end{aligned}
$$

where we extend the domain of definition of the Green's function to negative $k$-value by the definition $\tilde{G}_{n s}(\psi,-k)=$ $\tilde{G}_{n s}(\psi+\pi, k)$. Noting the Fourier variable $\xi=R \cos (\psi-\theta)$, we obtain

$$
G_{n s}(R, \theta)=\frac{1}{2 \pi} \int_{-\pi / 2}^{\pi / 2} \mathrm{~d} \psi \hat{G}_{n s}(\xi, \psi),
$$

where $\hat{G}_{n s}(\xi, \psi)$ is the one-dimensional Fourier transform of $|k| \tilde{G}_{n s}(k, \psi)$ along the $k$ axis. This one-dimensional Fourier transform is evaluated by FFT for every angle $\psi$ and the result is then fed back into Eq. (24) to evaluate the angular integral. It can also be remarked that discontinuities at the
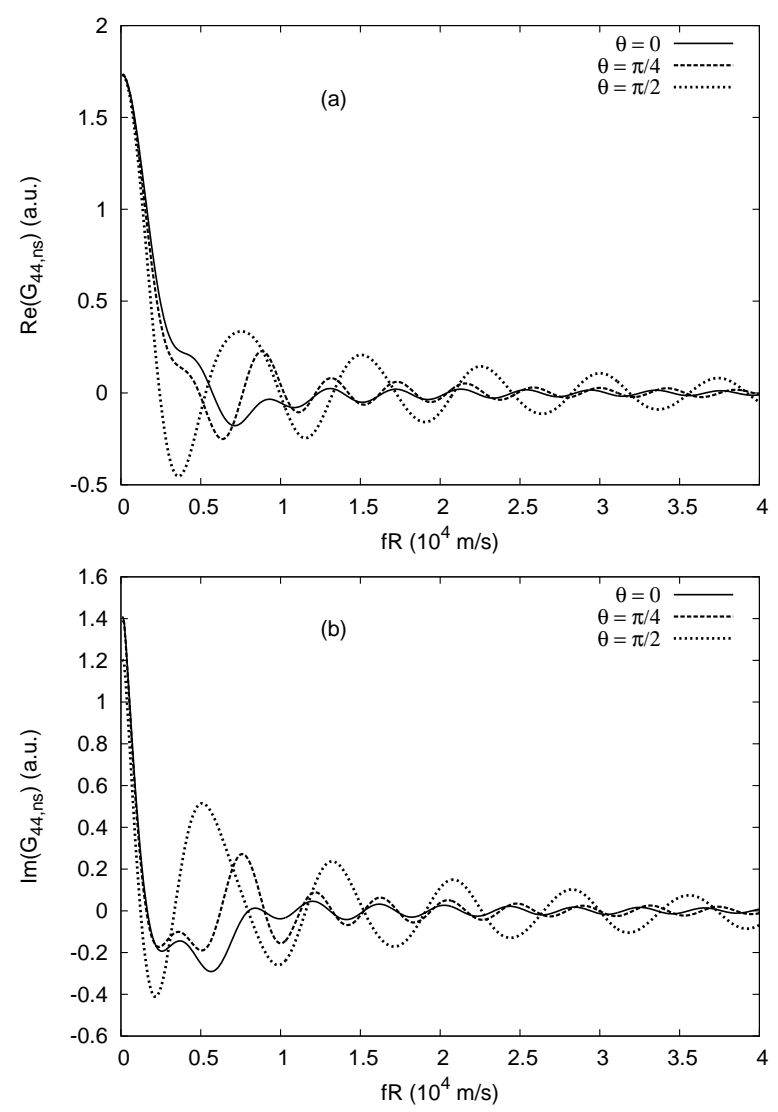

Fig. 9. Real (a) and imaginary (b) parts of $G_{44, n s}$, the non-singular contribution to the spatial surface Green's function $G_{44}$, for Y+128 lithium niobate.

origin as displayed by $\tilde{G}_{14}$ in Fig. 2 are naturally removed by multiplication with $|k|$.

Fig. 9 displays the non-singular contribution to the spatial surface Green's function $G_{44}$ for Y+128 lithium niobate half-space, again for three different propagation directions. It is mainly concentrated in the near field, with only the longitudinal LSAW giving rise to a pronounced oscillatory behavior for the propagation direction $\theta=\pi / 2$. Practically, 16384 uniformly distributed samples were used along the $k$ axis for the FFT and the integration was carried out from -0.02 to $0.02 \mathrm{~s} / \mathrm{m}$ in slowness units. The angular integral in Eq. (25) was evaluated with one sample per degree and using the trapezoid rule as the quadrature formula. It can be noted that the one-dimensional FFT can first be computed and stored for each required value of $\psi$, and then be used to obtain the value of $G_{n s}(R, \theta)$ for any observation point in an efficient manner.

Fig. 10 displays the imaginary part of the full surface Green's function of a half-space of $Y+128$ lithium niobate, i.e. the superposition of the contributions displayed in Figs. 6, 7 and 9.

\section{CONCLUSION}

We have discussed the computation of the two-dimensional harmonic spatial-domain Green's function at the surface of a piezoelectric half-space. The surface Green's function has 


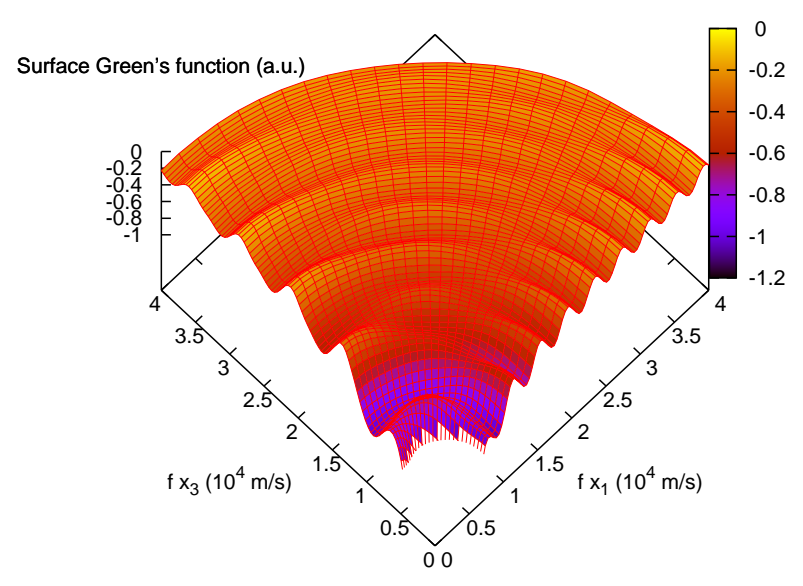

Fig. 10. Imaginary part of the spatial surface Green's function $G_{44}$ for a $\mathrm{Y}+128$ lithium niobate half-space. Only the first quadrant is displayed.

been expressed in polar coordinates which are the natural coordinates for a point-source problem. Starting from the known form of the Green's function expressed in the spectral domain, the various singular contributions are isolated and treated separately. It has been demonstrated that the surface acoustic wave contributions give rise to an anisotropic generalization of the Hankel function $H_{0}^{(2)}$, the spatial Green's function for the scalar two-dimensional wave equation. The surface acoustic wave contributions are dominant in the far field. The asymptotic behavior at infinity and at the origin (for the electrostatic contribution) have also been explicitely treated and shown to affect only the near field. The remaining non-singular part of the spectral Green's function has been obtained numerically by a combination of fast Fourier transform along the slowness axis and quadrature over the propagation direction. Illustrations have been given in the case of a Y+128 lithium niobate half-space for the $G_{44}$ Green's function that gives the electrical potential response of the surface to a point charge excitation. The same procedures are straightforwardly extended to purely mechanical or mixed electric-mechanical excitation problems. In addition to its application to the threedimensional simulation of SAW devices, we believe that the surface Green's function we have obtained will be useful in the description of surface acoustic waves in phononic band gap materials [28]-[30].

\section{REFERENCES}

[1] Y. Zhang, J. Desbois, and L. Boyer, "Characteristic parameters of surface acoustic waves in a periodic metal grating on a piezoelectric substrate," IEEE Trans. Ultrason., Ferroelec., Freq. Control, vol. 40, no. 3, pp. 183-192, 1993.

[2] R. C. Peach, "A general green function analysis for SAW devices," in IEEE Ultrason. Symp. Proc., 1995, pp. 221-225.

[3] H. P. Reichinger and A. R. Baghai-Wadji, "Dynamic 2D analysis of SAW devices including mass-loading," in IEEE Ultrason. Symp. Proc., 1992, pp. 263-266.

[4] H. P. Reichinger, A. R. Baghai-Wadji, and F. J. Seifert, "Stress pattern on the electrode/substrate interfaces in SAW-devices," in IEEE Ultrason. Symp. Proc., 1993, pp. 153-156.

[5] P. Ventura, J. Desbois, and L. Boyer, "A mixed FEM/analytical model of the electrode mechanical perturbation for SAW and PSAW propagation," in IEEE Ultrason. Symp. Proc., 1993, pp. 205-208.
[6] P. Ventura, J. M. Hodé, and M. Solal, "A new efficient combined FEM and periodic Green's function formalism for the analysis of periodic SAW structures," in IEEE Ultrason. Symp. Proc., 1995, pp. 263-269.

[7] G. Endoh, K. Hashimoto, and M. Yamaguchi, "Surface acoustic wave propagation characterization by finite element method and spectral domain analysis," Jpn. J. Appl. Phys., vol. 34, no. 5B, pp. 2638-2641, 1995.

[8] J. Koskela, J. P. Plessky, and M. M. Salomaa, "Suppression of the leaky SAW attenuation with heavy mechanical loading," IEEE Trans. Ultrason., Ferroelec., Freq. Control, vol. 45, no. 2, pp. 439-449, 1998.

[9] P. Ventura, J.-M. Hodé, J. Desbois, and M. Solal, "Combined FEM and Green's function analysis of periodic SAW structure, application to the calculation of reflection and scattering parameters," IEEE Trans. Ultrason., Ferroelec., Freq. Control, vol. 48, no. 5, pp. 1259-1274, 2001.

[10] P. Ventura, J. M. Hodé, and B. Lopes, "Rigorous analysis of finite saw devices with arbitrary electrode geometries," in IEEE Ultrason. Symp. Proc., 1995, pp. 257-262.

[11] J. Koskela, J. V. Knuuttila, P. T. Tikka, C. S. Hartmann, V. P. Plessky, and M. M. Salomaa, "Mechanism for acoustic leakage in surface-acoustic wave resonators on rotated y-cut lithium tantalate substrate," Appl. Phys. Lett., vol. 75, no. 17, pp. 2683-2685, 1999.

[12] G. Clark, R. F. Milsom, and J. Schofield, "3-D modal analysis of SAW filters," in IEEE Ultrason. Symp. Proc., 1985, pp. 26-30.

[13] K. Hashimoto, G. Endoh, M. Ohmaru, and M. Yamaguchi, "Analysis of SAWs obliquely propagating under metallic gratings with finite thickness," Jpn. J. Appl. Phys., vol. 35, no. 5B, pp. 3006-3009, 1996.

[14] M. Jungwirth, N. Pocksteiner, G. Kovacs, and R. Weigel, "Analysis of general multi-channel planar waveguides," IEEE Trans. Ultrason., Ferroelec., Freq. Control, vol. 49, no. 4, pp. 519-527, 2002.

[15] V. Laude and S. Ballandras, "Slowness curves and characteristics of surface acoustic waves propagating obliquely in periodic finite-thickness electrode gratings," J. Appl. Phys., vol. 94, no. 2, pp. 1235-1242, 2003.

[16] M. Solal, "A P-matrix based model for the analysis of SAW transversaly coupled resonator filters including guided modes and a continuum of radiated waves," IEEE Trans. Ultrason., Ferroelec., Freq. Control, vol. 50, no. 12, pp. 1729-1741, 2003.

[17] M. Solal, V. Laude, and S. Ballandras, "A p-matrix based model for SAW grating waveguides taking into account modes conversion at the reflection," IEEE Trans. Ultrason., Ferroelec., Freq. Control, vol. 51, no. 12, pp. 1690-1696, 2004.

[18] H. Zidek, A. Baghai-Wadji, and F. Seifert, "Full-wave 3D analysis of singly- and doubly periodic SAW-structures," in IEEE Ultrason. Symp. Proc., 1992, pp. 11-14.

[19] H. Zidek, A. Baghai-Wadji, and O. Männer, "Full-wave 3D analysis of wave scattering on SAW-structures with finite aperture," in IEEE Ultrason. Symp. Proc., 1993, pp. 149-152.

[20] A. H. Fahmy and E. L. Adler, "Propagation of surface acoustic waves in multilayers: A matrix description," Appl. Phys. Lett., vol. 22, no. 10, pp. 495-497, 1973.

[21] P. M. Smith, "Dyadic Green's function for multi-layer SAW substrates," IEEE Trans. Ultrason., Ferroelec., Freq. Control, vol. 48, no. 1, pp. 171-179, 2001

[22] T. Pastureaud, V. Laude, and S. Ballandras, "Stable scattering-matrix method for surface acoustic waves in piezoelectric multilayers," Appl. Phys. Lett., vol. 80, no. 14, pp. 2544-2546, 2002.

[23] J. G. Harris, Linear elastic waves. Cambridge: Cambridge University Press, 2001.

[24] P. M. Morse and H. Feshbach, Methods of theoretical physics. New York: McGraw-Hill, 1953, pp. 1335-1343.

[25] L. Boyer, J. Desbois, Y. Zhang, and J.-M. Hodé, "Theoretical determination of the pseudo surface acoustic wave characteristic parameters," in IEEE Ultrason. Symp. Proc. IEEE, 1991, pp. 354-358.

[26] E. L. Adler, "SAW and pseudo-SAW properties using matrix methods," IEEE Trans. Ultrason., Ferroelec., Freq. Control, vol. 41, no. 6, pp. 876-882, 1994.

[27] V. Laude, M. Wilm, and S. Ballandras, "A least-action principle for the estimation of the slowness and the attenuation of pseudo surface acoustic waves," J. Appl. Phys., vol. 93, no. 12, pp. 10084-10 088, 2003.

[28] T. Gorishnyy, C. K. Ullal, M. Maldovan, G. Fytas, and E. L. Thomas, "Hypersonic phononic crystals," Phys. Rev. Lett., vol. 94, p. 115501, 2005.

[29] V. Laude, M. Wilm, S. Benchabane, and A. Khelif, "Full band gap for surface acoustic waves in a piezoelectric phononic crystal," Phys. Rev. E, vol. 71, p. 036607, 2005.

[30] T.-T. Wu, L.-C. Wu, and Z.-G. Huang, "Frequency band-gap measurement of two-dimensional air/silicon phononic crystals using layered 
slanted finger interdigital transducers," J. Appl. Phys., vol. 97, p. 094916, 2005.

[31] I. S. Gradshteyn and I. M. Ryzhik, Table of Integrals, Series and Products, fifth edition. Academic Press, 1994.

\section{APPENDIX}

In this appendix, it is shown that the SAW contribution to the spatial Green's function, Eq. (12), reduces to $g_{s}(R, \theta)=$ $-\imath\left(k_{0}^{2} a_{i} / 4\right) H_{0}^{(2)}\left(k_{i} R\right)$ in case of isotropic SAW propagation. Substituting $k_{s}(\psi)=k_{i}$ and $a_{s}(\psi)=a_{i}$ in Eq. (12) we have

$$
\begin{aligned}
G_{s}(R, \theta)= & \frac{k_{0}^{2} a_{i}}{(2 \pi)^{2}}\left(2 \int_{-\pi / 2}^{\pi / 2} \mathrm{~d} \psi h\left(k_{i} R \cos \psi\right)\right. \\
& \left.-\imath \pi \int_{-\pi / 2}^{\pi / 2} \mathrm{~d} \psi \exp \left(-\imath k_{i} R \cos \psi\right)\right) .
\end{aligned}
$$

Now we have

$$
\begin{array}{r}
\int_{-\pi / 2}^{\pi / 2} \mathrm{~d} \psi h\left(k_{i} R \cos \psi\right) \\
=\int_{0}^{\infty} \frac{y \mathrm{~d} y}{1+y^{2}} \int_{-\pi / 2}^{\pi / 2} \mathrm{~d} \psi e^{-y k_{i} R \cos \psi} \\
=\int_{0}^{\infty} e^{-y_{1} k_{i} R} \mathrm{~d} y_{1} \int_{-\infty}^{\infty} \frac{\mathrm{d} y_{3}}{1+y_{1}^{2}+y_{3}^{2}} \\
=\int_{0}^{\infty} e^{-y_{1} k_{i} R} \mathrm{~d} y_{1} \times \frac{\pi}{\sqrt{1+y_{1}^{2}}} \\
=\pi \int_{0}^{\infty} \mathrm{d} t \exp \left(-k_{i} R \sinh t\right)
\end{array}
$$

where use has been made of identity (3.111) in Ref. [31]. In addition, we have

$$
\int_{-\pi / 2}^{\pi / 2} \mathrm{~d} \psi e^{-\imath k_{i} R \cos \psi}=\int_{0}^{\pi} \mathrm{d} \psi e^{-\imath k_{i} R \sin \psi} .
$$

Remembering the following integral representations of Bessel and Neumann functions (formulas (8.411) and (8.415.4) in Ref. [31])

$$
\begin{aligned}
J_{0}(x) & =\frac{1}{\pi} \int_{0}^{\pi} \cos (x \sin \theta) \mathrm{d} \theta \\
N_{0}(x) & =\frac{1}{\pi} \int_{0}^{\pi} \sin (x \sin \theta) \mathrm{d} \theta-\frac{2}{\pi} \int_{0}^{\infty} \mathrm{d} t e^{-x \sinh t}(30)
\end{aligned}
$$

and the definition of the Hankel function of second kind and zero-th order, $H_{0}^{(2)}(x)=J_{0}(x)-\imath N_{0}(x)$, we finally obtain

$$
g_{s}(R, \theta)=-\imath\left(k_{0}^{2} a_{i} / 4\right) H_{0}^{(2)}\left(k_{i} R\right) .
$$

